\title{
Thermal Effect and Quenching of Exciplex Fluorescence of Polystyrene Derivatives by Dimethyl Terephthalate in Solid Films
}

\author{
Afrah E. Ramadhan, ${ }^{1}$ R. K. Ahmed,${ }^{2}$ and Khalid E. AL-AnI ${ }^{3, \dagger}$ \\ ${ }^{1}$ Department of Industrial Chemistry, Institute of Technology, Baghdad, Iraq \\ ${ }^{2}$ Department of Physics, Mustinseriah University, Baghdad, Iraq \\ ${ }^{3}$ Department of Chemistry, Faculty of Science, Hashemite University, Zarka, Jordan
}

(Received June 15, 2005; Accepted November 23, 2005; Published April 15, 2006)

\begin{abstract}
The effects of added dimethyl terephthalate to the fluorescence emission of poly(4-ethoxystyrene), poly(4-methoxystyrene), poly(4-methoxy $\alpha$-methyl styrene) and poly(4-methoxyphenyl-styrene) were studied in solid films and in dichloromethane solution. A quenching of the excimer emission was accompanied with the appearance of a strong exciplex emission in solid films. In dichloromethane solution, only quenching of the excimer emission was noticed, but no exciplex emission was detected. Increase in the temperature of solutions or solid films of these polymers caused a decrease in the intensity of exciplex and an increase in the polymer emissions. This thermal effect was attributed to the thermal distraction of the formed exciplex chromophores that can enhance excimer emission. The thermostability of these polymers depends on electronic nature of substituent and their ability to stabilize radicals forming on chain scission. A kinetic model is proposed to account for these observations concerning the singlet excited state of polymers. The activation energies of intermolecular exciplex formation were calculated.

[DOI 10.1295/polymj.38.355]

KEY WORDS Thermal Effect / Polystyrene Derivatives / Fluorescence Quenching / Thermal Quenching /
\end{abstract}

An exciplex is a molecular complex of definite stoichiometry which is stable only in the excited state. A number of studies were made on the fluorescence of exciplexes that formed between different substituted aromatic hydrocarbons and amines. ${ }^{1-4}$ The fluorescence of poly(1-vinylcarbazole) in solution or solid film was diminished upon the addition of dimethyl terephthalate. ${ }^{5}$ The fluorescence quenching of poly(1-vinylcarbazole) is accompanied by the appearance of a broad red-shifted structure less band, and is attributed to the emission of an exciplex chromophores.

The formation of an intermolecular exciplex and the electron transfer processes were studied in vinyl copolymers, doped with $N, N$-dimethylaniline and cyanobenzene moieties as pendant groups. ${ }^{3}$ In the formation of exciplex, the intramolecular dominated the intermolecular process, even in the presence of strong acceptor. The exciplex formation in 9-vinylphenanthrene- $N, N$-dimethyl aminostyrene copolymers and the electron transfer process were studied in polar solvents. Intensities of the exciplex fluorescence which kept unchanged regardless of the copolymer compositions led to speculate that the efficient energy migration takes place from an excited phenanthrene unit to an exciplex forming site on the polymer chain. ${ }^{6}$

The addition of dimethyl terephthalate to the solid films causes quenching of the excimer fluorescence of both poly(4-methoxystyrene) and poly(4-ethoxystyrene). ${ }^{7}$ New fluorescence bands were observed at about 440 and $446 \mathrm{~nm}$ for (4-methoxystyrene) and poly(4-ethoxystyrene) respectively, and were assigned as an exciplex fluorescence. The Stern-Volmer plots of the excimer quenching of vinyl polymers ${ }^{8,9}$ showed a deviation from linearity of polystyrene. This cannot be accounted by the assumption that the excimer is quenched by direct quenching as well as by quenching of a precursor state, the excited monomer singlet.

There have been many studies conducted on the thermal degradation of polystyrene, ${ }^{10-12}$ and on substituted polystyrenes. ${ }^{13-16}$ Depolymerization to monomer is generally an important mode of decomposition for both polystyrene and substituted polystyrenes although this is not always the case since, for example, poly(4-nitrostyrene) does not yield monomer. In this case ring decomposition will still occur. The thermal degradation of a series of para-substituted polystyrenes has been studied in isothermal conditions using pyrolysis chromatography and in dynamic conditions using thermo gravimetry. The pyrolysis of these substituted polystyrenes gives monomer as the main product for all polymers. The other distribution of products supports the view that thermo destruction

${ }^{\dagger}$ To whom correspondence should be addressed (Tel: +962 05390 3333/ext: 4149; Fax: +962 05390 3349, E-mail address: khalid7@hu.edu.jo; khalider44@yahoo.com). 
Table I. Absorption and fluorescence parameters for polystyrene derivatives in DCM

\begin{tabular}{lccrcc}
\hline \multicolumn{1}{c}{ Polymer } & $T_{\mathrm{g}}{ }^{\mathrm{b}}$ & $M_{\mathrm{w}}$ & $M_{\mathrm{n}}$ & $\begin{array}{c}\lambda_{\max } \\
\text { Excitation } \\
(\mathrm{nm})\end{array}$ & $\begin{array}{c}\lambda_{\max } \\
\text { Emissions } \\
(\mathrm{nm})^{\mathrm{a}}\end{array}$ \\
\hline Poly(4-methoxystyrene) & $360 \pm 5$ & 21500 & 9000 & 277 & 295,346 \\
Poly(4-ethoxystyrene) & $356 \pm 5$ & 32500 & 23500 & 278 & 291,348 \\
Poly(4-methoxyphenylstyrene) & $403 \pm 5$ & 10000 & 4500 & 273 & 310 \\
Poly(4-methoxy- $\alpha$-methylstyrene) & $380 \pm 5$ & 24000 & 10000 & 275 & 300 \\
\hline
\end{tabular}

${ }^{\text {a }}$ First $\lambda_{\max }$ emission corresponds to monomer emission and the second $\lambda_{\max }$ emission coresponds to excimer emission. ${ }^{b}$ Data are from ref 18.

of these polymers starts from the random scission. ${ }^{17}$ The main product of this process is the monomer while the thermal stability of the alkylated polystyrene is somewhat higher than that of polystyrene.

The objective of the present paper is to study the exciplex formation in para-substituted polystyrenedimethyl terephthalate system in solid films and the thermal effect on exciplex and excimer fluorescence emissions from these polymers.

\section{EXPERIMENTAL}

\section{Materials}

Substituted polystyrenes were synthesized by thermal free radical polymerization in solution using $\alpha, \alpha^{\prime}$-azobisisobutyronitrile (ABIN), as initiators by a previously described procedure. ${ }^{18}$ They were reprecipitated several times into methanol and found to have a viscosity average molecular weight as presented in Table I. Dimethyl terephthalate (DMT) was purchased from Fluka GMBH; moreover, it was recrystallized several times in benzene and vacuum sublimed. Spectroscopic-quality, dichloromethane (DCM), tetrahydrofuran, benzene and acetonitrile were found to give no detectable emission when excited in the range $250-400 \mathrm{~nm}$. These solvents were purchased from Fluka GMBH and were used as they were received.

\section{Absorption and Fluorescence Measurements}

The measured absorption spectra of the polymers in DCM and other used solvents were obtained using a "Cary 100 Bio UV-Vis Spectrophotometer." To record fluorescence spectra of polymers in solid films and in dichloromethane, samples were excited with 260-280 nm, and measured on a FS 900 CDT steady state spectrofluorometer from Edinburgh Analytical Instruments (EAI).

\section{Solid Films Preparation}

The preparation of the thin-doped polymer solid films proceeded in the following manner: a microbalance was used for weighing about 100-200 mg sample of the polymers, and then dissolved in dichloro- methane. The concentration of the energy acceptor (DMT) in each film was too low to make it possible to weigh this material for each individual film. Consequently, a stock solution of the acceptor in dichloromethane solvent was prepared, and then from which solid films were prepared. The variation in acceptor concentration was made by dilution of the stock solution and the concentration in each film was calculated from the volume of solution added to the polystyrene derivative-DMT solid mixture. Films were then cast from solution by placing a small amount onto a carefully cleaned $(0.2 \times 20 \times 20 \mathrm{~mm})$ fused quartz plate. The solvent was slowly evaporated by placing the quartz plate in a covered Pyrex Petri dish in a uniform temperature environment. The resulting films appeared to be of uniform thickness and were optically clear. No effort was made to determine film thickness or to keep a constant thickness, since this parameter had no apparent effect on the experimental results after its removal from the plate. The film was dried at $50^{\circ} \mathrm{C}$ in an oven for a few minutes, and then was pumped under vacuum in order to eliminate any residual solvent. ${ }^{19,20}$

\section{RESULTS AND DISCUSSION}

\section{Absorption and Steady State Fluorescence Spectra}

Figure 1, shows the absorption spectra for PMOS, PEOS and poly(4-methoxyphenyl-styrene) PMPS in solid films, with maximum absorption at 278, 277 and $275 \mathrm{~nm}$ respectively. According to these spectra, only one absorption band appears, this indicate that mixing of the electronic ground state chromophores with neighboring chromophores is weak. Absorption and fluorescence spectra for PMOS and PEOS were previously reported and no appreciable ground state interaction was observed between these polymers and DMT moieties. ${ }^{18}$ Excitation was preformed at wavelength greater than $267 \mathrm{~nm}$, where DMT did not absorb and only polymer chromophores were excited. For PMOS, PEOS, PMPS and PMMS as acceptors, ground state CT complex formation do not occur to any appreciable extent with the used donor DMT. Exciplexes are formed via a bimolecular quenching of 


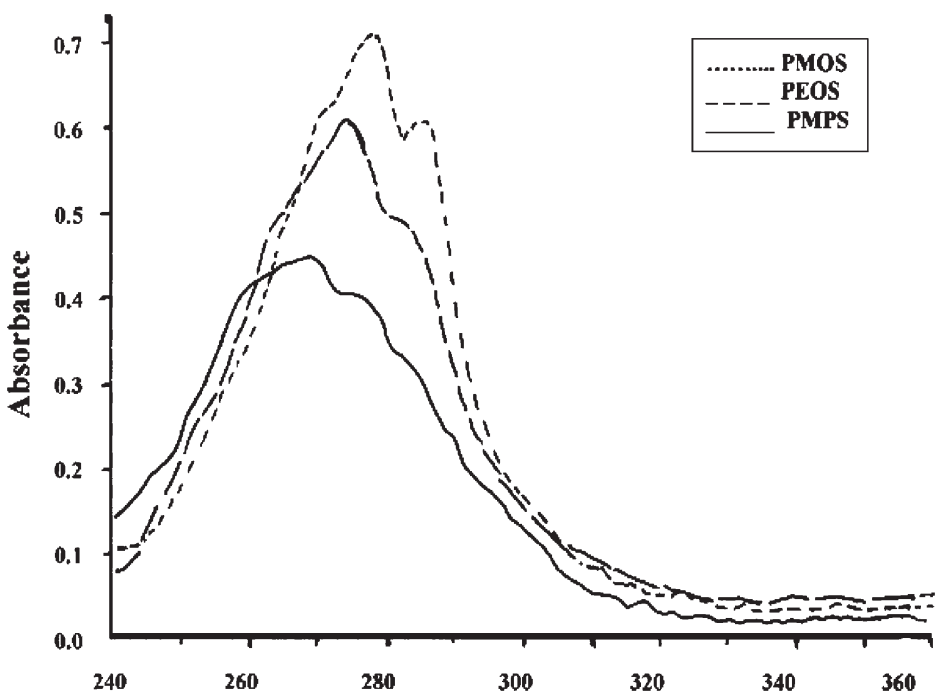

Figure 1. UV-absorption spectra for poly (para-substituted styrene) in solid films, film thickness $(0.1 \pm 0.02 \mathrm{~mm})$.

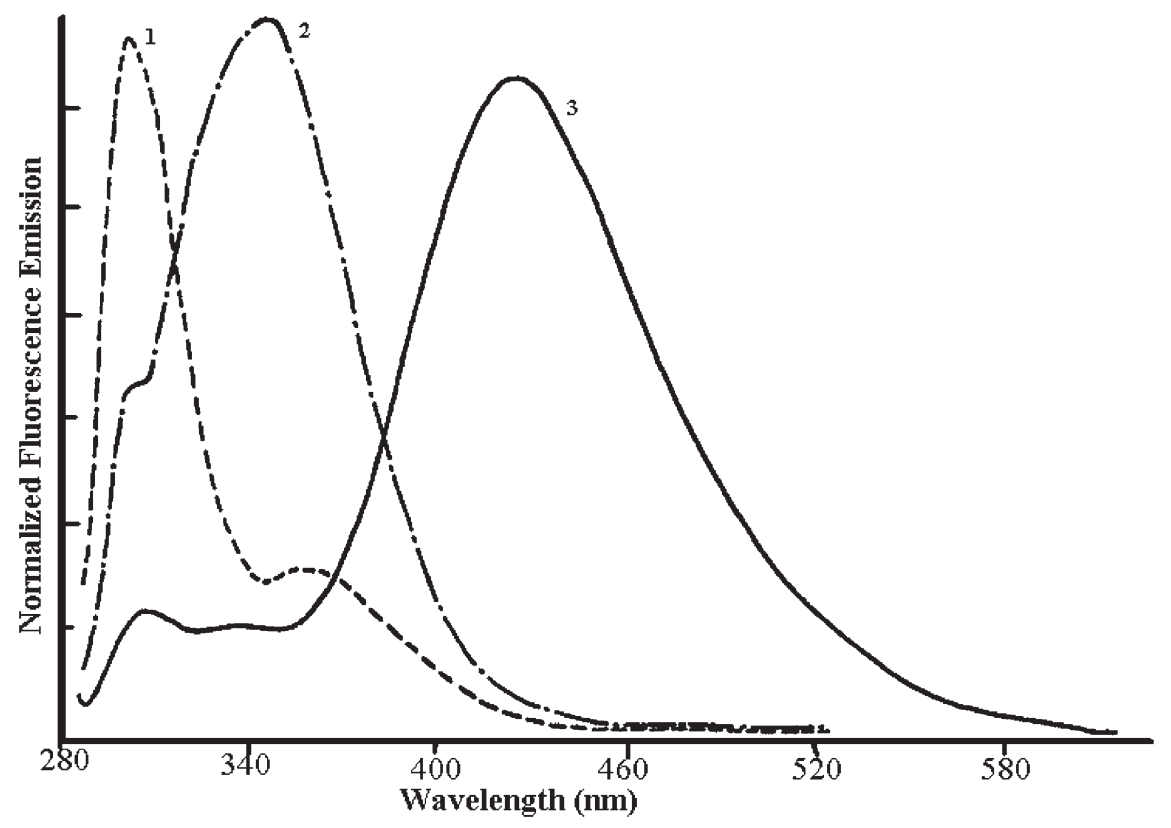

Figure 2. Corrected fluorescence spectra of poly(4-ethoxystyrene), 1: in dichloromethane solution at concentration (0.0001 M), 2: in solid film, and 3: in 3\% w/w doped dimethyl terephthalate at excitation wavelength of $280 \mathrm{~nm}$.

the excited polymer chromophores and not via excitation in the CT absorption bands of the ground state (polymer-DMT) complexes.

In a recent work, ${ }^{18}$ PEOS and PMOS, gave strong excimer fluorescence and a relatively weak fluorescence for the monomer in solid films. Poly(4-methoxy $\alpha$-methylstyrene) PMMS and poly(4-methoxyphenylstyrene) PMPS, gave only monomer fluorescence in solid films due to the size of the substituents which made it impossible that two rings approach near enough to form excimers. Figure 2 shows the fluorescence emissions for PEOS in dichloromethane solution in casted dichloromethane solid film and in 3\% $\mathrm{w} / \mathrm{w}$ doped dimethyl terephthalate at exciting wavelength of $280 \mathrm{~nm}$. The absence of monomeric emis- sion in PEOS solid film compared to that in DCM solution is attributed to the absence of solvent molecules in one side and to the low activation energy for excimer formation in favorable sites. ${ }^{18}$ Furthermore, as one expects, the chain conformations in solid films of PMOS and PEOS do not undergo collision deactivation by solvent molecules. These observations are very close to what has been obtained in vinyl polymers. ${ }^{21}$ For PMMS and PMPS, only monomer fluorescence is observed in DCM solution and solid films. This may arise either from a small barrier to excimer formation for favorable sits, or from a partial thermal relaxation of the chain segments which are more loosely bonded in solvent medium. 


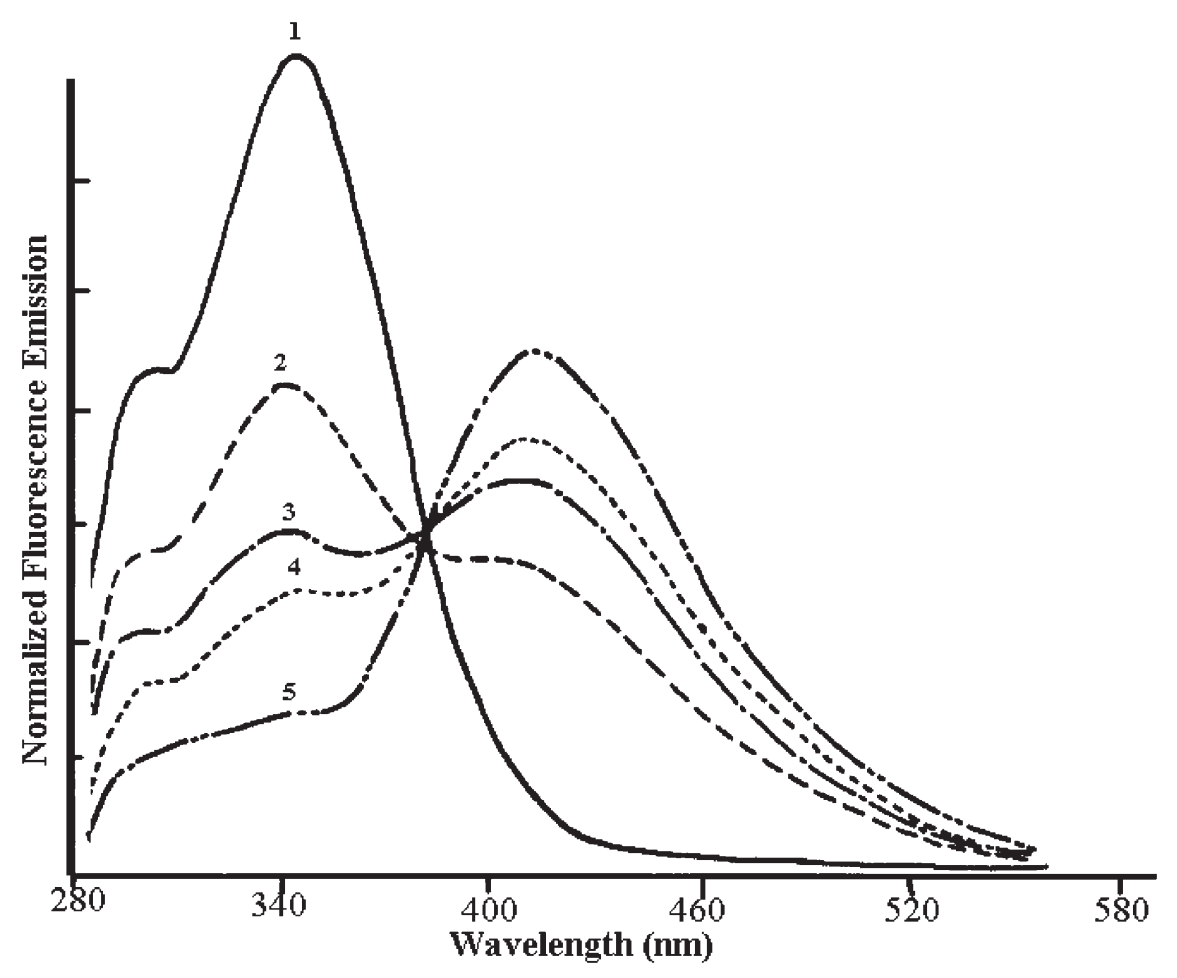

Figure 3. Corrected fluorescence spectra of poly(4-methoxy styrene) films doped with dimethyl terephthalate at 1: $0.0 \%, 2: 1.0 \%, 3$ : $1.5 \%, 4: 2.0 \%$ and $5: 3.0 \%$, at excitation wavelength of $280 \mathrm{~nm}$.

\section{Fluorescence Quenching}

The fluorescence quencher DMT was chosen as the acceptor, because its lowest lying singlet state falls considerably lower in energy than that of polystyrene (PS) and polystyrene derivatives (PSD). Thus, it can quench PSD fluorescence through a single step longrange energy transfer by the foster dipole-dipole inductive resonance mechanism. It was determined however that in solid films and even in fluid solutions, DMT quench PSD fluorescence at a rate corresponding to the diffusion limit with the mechanism involving a charge transfer interaction (CT) between the electronically excited PSD and the ground state DMT. The emitting (CT) complexes are formed via a bimolecular reaction of the uncomplexed excited donor (polymer) and the ground state acceptor (DMT), and not via excitation in the (CT) absorption band of the ground state (polymer-DMT) complex. The addition of increasing amount of DMT would result in decreasing excimer and monomer emissions from excited PSD and increasing the fluorescence intensity of a longer range band of the exciplex emission, as shown for PEOS as in Figure 2. However, the fluorescence lifetimes of PSD would remain constant since only those excited PSD molecules which have a DMT molecule within some critical interaction radius would be quenched, while those without the DMT molecules would give fluorescence with their unperturbed lifetime. ${ }^{19}$ The addition of relatively high concentration of DMT to polystyrene derivatives in di- chloromethane, benzene, tetrahydrofuran and acetonitrile did not show any exciplex fluorescence. In contrast, all used polymers exhibited exciplex emission upon the addition of DMT concentration in dichloromethane cast solid films, as shown in Figure 3 for PEOS. The intensity of exciplex fluorescence increases with increasing DMT concentration. For the PEOS-DMT system, an isosbestic point is observed at $390 \mathrm{~nm}$ which appears to indicate that there is only one new species formed with wavelength of maximum emission around $424 \mathrm{~nm}$. The $3 \%$ by mass of added DMT was enough to quench most of the excimeric emission in solid films.

From Figure 3, it was noticed that the emission band at the longer wavelength is due to the exciplex formed between the excited PSD and DMT. This is easily confirmed by the excitation spectrum of the new emission band which shows good similarity with the absorption spectra of PSD in solid films. ${ }^{22}$ In PSD-DMT systems, two processes are conceivable for the formation of exciplex. One is the direct energy transfer process from the electron donor DMT to the excited polystyrene derivatives, as in the case of PMMS and PMPS; the other is the energy transfer from the electron donor DMT to the formed excimer, as in case of PMOS and PEOS. Furthermore a good estimate for the energy of the charge transfer state of the complex between excited polymer chromophores and ground state DMT is obtained by comparison of the oxidation potential of the donor DMT with 


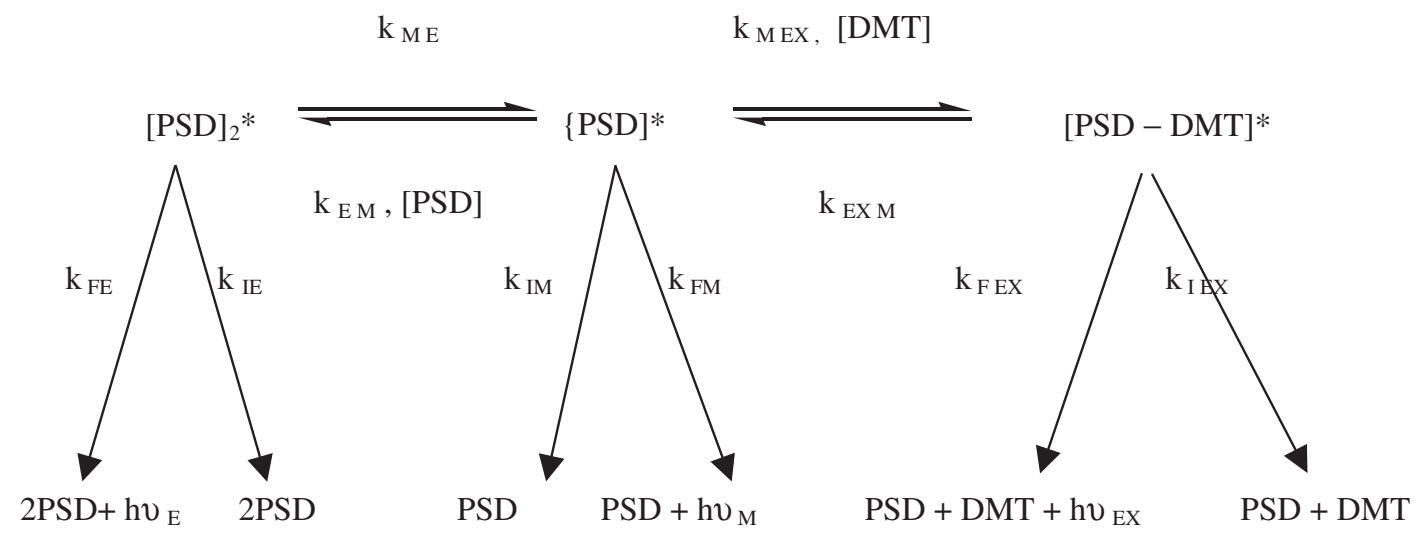

Scheme 1.

the reduction potential of the acceptor (polymer chromophore). Unfortunately, these data are not available to us at this time nor reported in the literature.

The quenching of the [PSD-DMT]* system can be described by the application of exciplex kinetics (Scheme 1, after Birks), ${ }^{23}$ and will be discussed first. [PSD-DMT]* represents the exciplex formed from excited PSD* and ground state DMT. [PSD] ${ }_{2}^{*}$ represents the excimer formed from excited PSD* and ground state PSD. The rate constants $k_{\mathrm{FM}}, k_{\mathrm{FE}}$ and $k_{\mathrm{FEX}}$ are those for radiative decay of free PSD*, the excimer
$[\mathrm{PSD}]_{2}^{*}$ and the exciplex [PSD-DMT] ${ }^{*}$ respectively. The rate constant $k_{\mathrm{IM}}, k_{\mathrm{IE}}$ and $k_{\mathrm{IEX}}$ are those for non-radiative decay of $\mathrm{PSD}^{*},[\mathrm{PSD}]_{2}{ }^{*}$ and $[\mathrm{PSD}-$ $\mathrm{DMT}^{*}$ respectively. Finally, $k_{\mathrm{MEX}}$ is the rate constant for exciplex formation, while $k_{\mathrm{ME}}$ is the rate constant for excimer formation and the rate constant for the feedback step to give PSD* and DMT is $k_{\mathrm{EXM}}$.

Appling the steady-state approximation to the concentration of monomer [PSD*], the excimer $[\mathrm{PSD}]_{2}{ }^{*}$ and to the exciplex [PST-DMT]* gives:

$$
\begin{aligned}
& {[\mathrm{PSD}]^{*}=\frac{I a k_{\mathrm{E}} k_{\mathrm{EX}}}{k_{\mathrm{EX}}\left(k_{\mathrm{M}} k_{\mathrm{E}}-k_{\mathrm{EM}} k_{\mathrm{ME}}\right)+k_{\mathrm{E}} k_{\mathrm{MEX}}\left(k_{\mathrm{EX}}-k_{\mathrm{EXM}}\right)[\mathrm{DMT}]}} \\
& {\left[\operatorname{IasD}_{2}{ }^{*}=\frac{k_{\mathrm{MEX}} k_{\mathrm{EX}}}{k_{\mathrm{EX}}\left(k_{\mathrm{M}} k_{\mathrm{E}}-k_{\mathrm{EM}} k_{\mathrm{ME}}\right)+k_{\mathrm{E}} k_{\mathrm{MEX}}\left(k_{\mathrm{EX}}-k_{\mathrm{EXM}}\right)[\mathrm{DMT}]}\right.} \\
& {[\mathrm{PSD}-\mathrm{DMT}]^{*}=\frac{I a k_{\mathrm{MEX}} k_{\mathrm{E}}}{k_{\mathrm{EX}}\left(k_{\mathrm{M}} k_{\mathrm{E}}-k_{\mathrm{EM}} k_{\mathrm{ME}}\right)+k_{\mathrm{E}} k_{\mathrm{MEX}}\left(k_{\mathrm{EX}}-k_{\mathrm{EXM}}\right)[\mathrm{DMT}]}}
\end{aligned}
$$

Where $k_{\mathrm{M}}=\left(k_{\mathrm{FM}}+k_{\mathrm{IM}}+k_{\mathrm{EM}}\right), k_{\mathrm{E}}=\left(k_{\mathrm{FE}}+k_{\mathrm{IE}}+k_{\mathrm{EM}}\right)$ and $k_{\mathrm{EX}}=\left(k_{\mathrm{FEX}}+k_{\mathrm{IEX}}+k_{\mathrm{EXM}}\right)$.

$I_{[\mathrm{PSD}]^{*}}, I_{[\mathrm{PSD}] 2^{*}}$ and $I_{[\mathrm{PSD}-\mathrm{DMT}]^{*}}$, the intensities of monomer, excimer and exciplex fluorescence are given by:

$$
I_{(\mathrm{PSD})^{*}}=k_{\mathrm{FM}}[\mathrm{PSD}]^{*}, I_{(\mathrm{PSD}) 2^{*}}=k_{\mathrm{FE}}[\mathrm{PSD}]_{2}{ }^{*} \text { and } I_{[\mathrm{PSD}-\mathrm{DMT}]^{*}}=k_{\mathrm{FEX}}[\mathrm{PSD}-\mathrm{DMT}]^{*}
$$

Replacement of $[\mathrm{PSD}]^{*},[\mathrm{PSD}]_{2}{ }^{*}$ and $[\mathrm{PSD}-\mathrm{DMT}]^{*}$ by their values from eq 1, 2 and 3 gives:

$$
\begin{aligned}
& I_{[\mathrm{PSD}]^{*}}=\frac{I a k_{\mathrm{FM}} k_{\mathrm{E}} k_{\mathrm{EX}}}{k_{\mathrm{EX}}\left(k_{\mathrm{M}} k_{\mathrm{E}}-k_{\mathrm{EM}} k_{\mathrm{ME}}\right)+k_{\mathrm{E}} k_{\mathrm{MEX}}\left(k_{\mathrm{EX}}-k_{\mathrm{EXM}}\right)[\mathrm{DMT}]} \\
& I_{[\mathrm{PSD}]_{2}}{ }^{*}=\frac{I a k_{\mathrm{FE}} k_{\mathrm{ME}} k_{\mathrm{EX}}}{k_{\mathrm{EX}}\left(k_{\mathrm{M}} k_{\mathrm{E}}-k_{\mathrm{EM}} k_{\mathrm{ME}}\right)+k_{\mathrm{E}} k_{\mathrm{MEX}}\left(k_{\mathrm{EX}}-k_{\mathrm{EXM}}\right)[\mathrm{DMT}]} \\
& I_{[\mathrm{PSD}-\mathrm{DMT}]^{*}}=\frac{I a k_{\mathrm{FEX}} k_{\mathrm{MEX}} k_{\mathrm{E}}}{k_{\mathrm{EX}}\left(k_{\mathrm{M}} k_{\mathrm{E}}-k_{\mathrm{EM}} k_{\mathrm{ME}}\right)+k_{\mathrm{E}} k_{\mathrm{MEX}}\left(k_{\mathrm{EX}}-k_{\mathrm{EXM}}\right)[\mathrm{DMT}]}
\end{aligned}
$$

From the above equations, we can write:

$$
\begin{aligned}
\frac{I_{[\mathrm{PSD}]^{*}}}{I_{[\mathrm{PSD}-\mathrm{DMT}]^{*}}} & =\frac{k_{\mathrm{FM}} k_{\mathrm{EX}}}{k_{\mathrm{FEX}} k_{\mathrm{MEX}}[\mathrm{DMT}]} \\
\frac{I_{[\mathrm{PSD}]^{2} 2}}{I_{[\mathrm{PSD}-\mathrm{DMT}]^{*}}} & =\frac{k_{\mathrm{FE}} k_{\mathrm{ME}} k_{\mathrm{EX}}}{k_{\mathrm{FEX}} k_{\mathrm{MEX}} k_{\mathrm{E}}[\mathrm{DMT}]}
\end{aligned}
$$

$$
\frac{I_{[\mathrm{PSD}]^{*}}}{I_{[\mathrm{PSD}]^{* 2}}}=\frac{k_{\mathrm{FM}} k_{\mathrm{E}}}{k_{\mathrm{FE}} k_{\mathrm{ME}}}
$$

In order to study the efficiency of the formed exciplex in these four polymers upon the addition of DMT, $I[\mathrm{PSD}]_{2}{ }^{*} / I[\mathrm{PSD}-\mathrm{DMT}]^{*}$ was plotted against 
w/w percentage of doped DMT in the cast films as shown in Figure 4. I[PSD-DMT] ${ }^{*}$ is the intensity of exciplex fluorescence and $I[\mathrm{PSD}]_{2}{ }^{*}$ is the intensity of excimer fluorescence. The intensity of exciplex

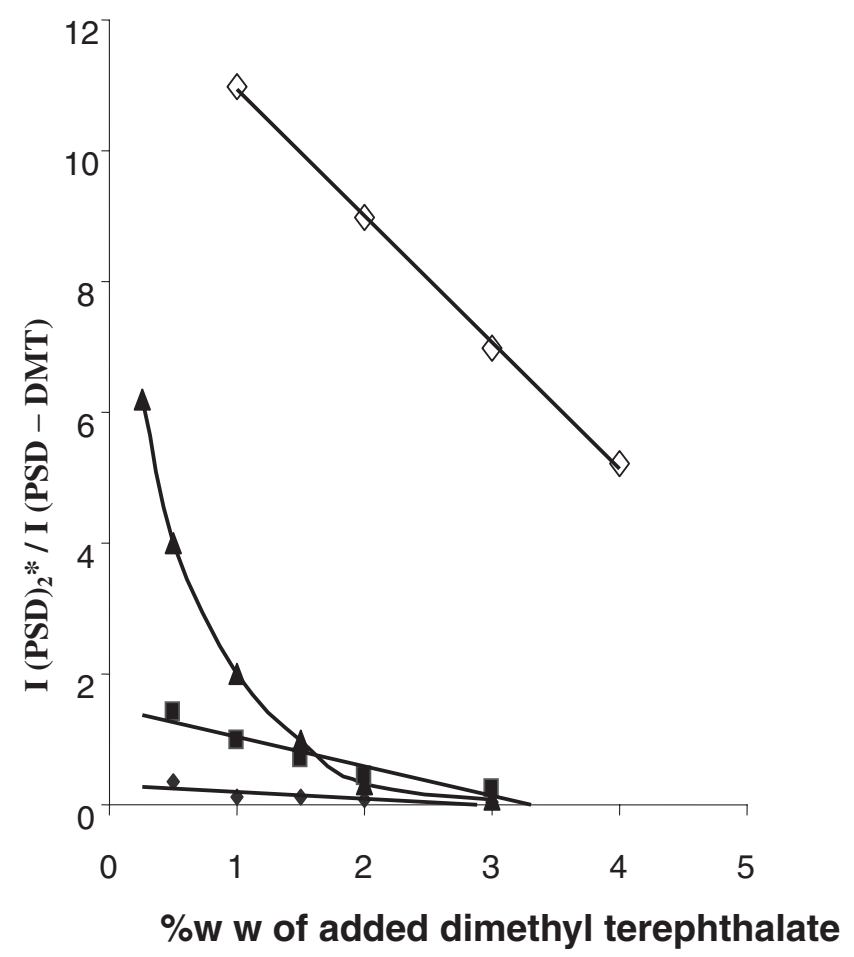

Figure 4. Effect of doped DMT (w/w \%) on $\left[I(\mathrm{PSD})^{*} /\right.$ $I$ (PSD-DMT)* $^{*}$ for $\boldsymbol{\Delta}$ (PMPS), $\boldsymbol{\square}(\mathrm{PEOS}), \boldsymbol{( P M O S )}$ and $\square$ (PMMS) in DCM cast films at excitation wavelength of 267, 268,273 and $275 \mathrm{~nm}$ respectively. fluorescence in [PMOS-DMT]* and [PEOS-DMT]* systems are almost the same. This indicates that almost the same effect can be obtained by the para substitution of both groups in polymer structure. The exciplex formation in [PMMS-DMT]* and [PMPS$\mathrm{DMT}^{*}$ systems was weak compared to the other two polymers. This may be attributed to the time required for folding of the molecule to bring the two chromophores close to each other, which seems too long compared with the excited lifetime. ${ }^{20}$ The stability of the exciplex formation is usually dictated by the extent of charge transfer interactions. For strongly bonded exciplexes, reverse dissociation $k_{\mathrm{EXM}}$ is very small and the fluorescence properties of the exciplex will depend upon the rate constant $k_{\mathrm{IEX}}$ relative to $k_{\mathrm{FEX}}$. The fluorescence quenching of poly(4-methoxystyrene) did not give a straight line on the SternVolmer plot but gave a very strong quenching efficiency compared with the other three polymers. This may be attributed to the strong rate constant $k_{\mathrm{MEX}}$ relative to $k_{\mathrm{FEX}}$.

\section{Thermal Quenching of Fluorescence}

Emission spectra for all these polymers are quite temperature dependent, while the absorption spectra show no significant change over the temperature range (293-393 K). The only noticed change is narrowing the vibration structure at lower temperatures. Figure 5 , shows the temperature dependence of emission spectra for PEOS films cast in DCM containing 3\% by mass of DMT. With increasing temperature, the

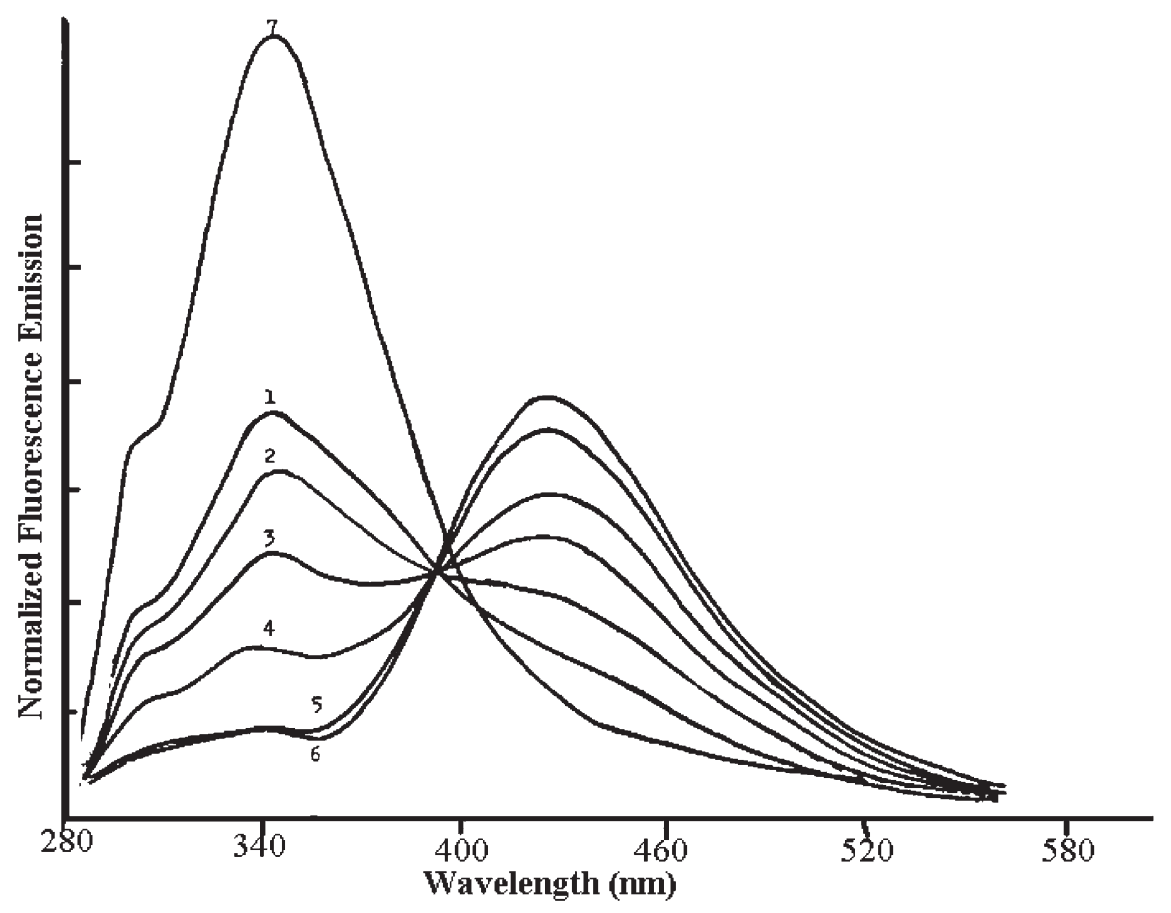

Figure 5. Corrected fluorescence spectra of poly(4-ethoxystyrene) films cast in DCM containing 3\% w/w DMT at 1: $373 \mathrm{~K}, 2: 363 \mathrm{~K}$, 3: $353 \mathrm{~K}, 4: 333 \mathrm{~K}, 5: 313 \mathrm{~K}$ and 6: $273 \mathrm{~K}$, compared with spectrum (7) of (PEOS) film only. 


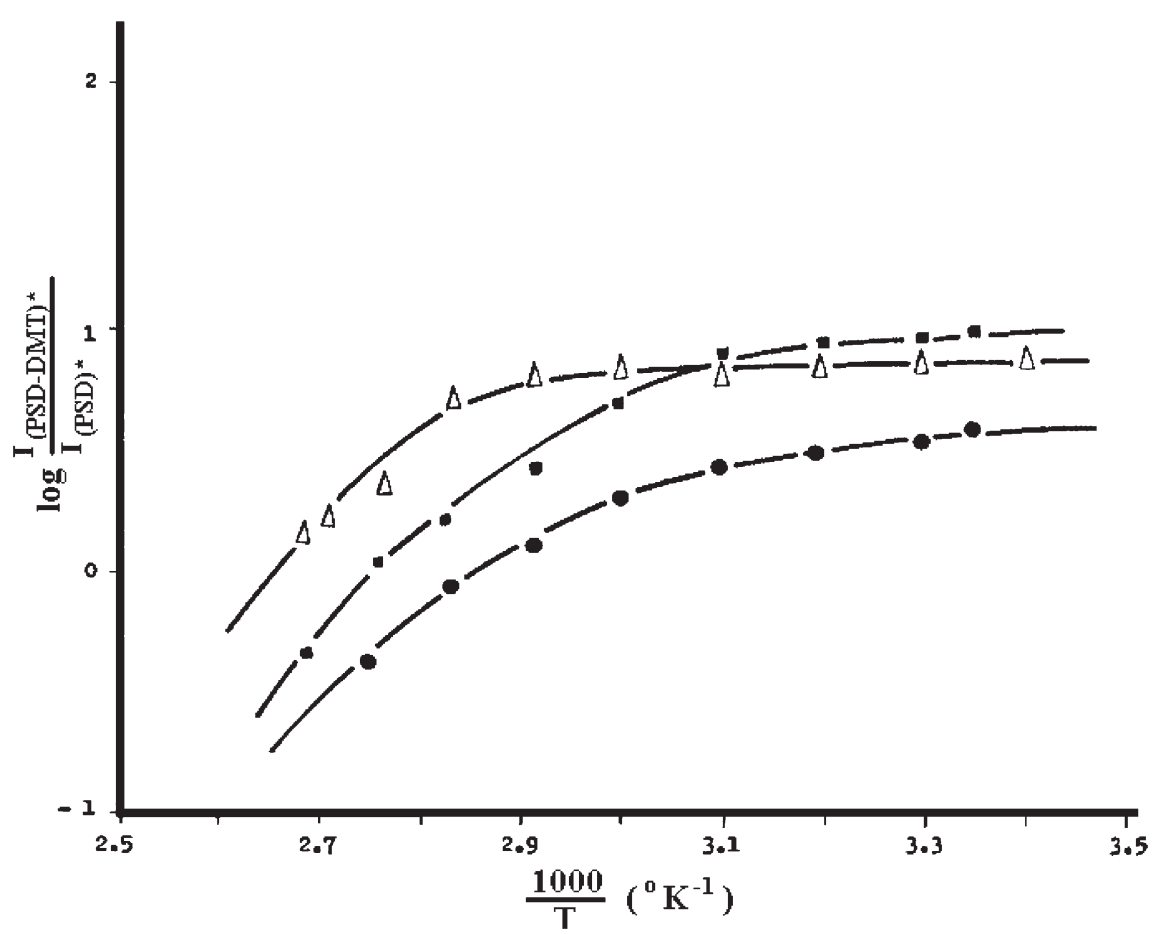

Figure 6. Temperature dependence of the exciplex emission and excimer emission on temperature, on $\triangle(\mathrm{PEOS}+3 \% \mathrm{DMT})$ and $\bullet(\mathrm{PMOS}+2 \% \mathrm{DMT}) . \log \frac{I_{[\mathrm{[PSD}-\mathrm{DMT})^{*}}}{I_{[\mathrm{PSD}]^{*}}}$ values are plotted against 1/T.

intensity of the exciplex emission decreases with the resultant increase in the monomer emission intensity as shown in Figure 5. A formation of an isoemissive point at $396 \pm 5 \mathrm{~nm}$ was also observed. Similar temperature dependence has been reported for the exciplex formation of 9-cyano,10-ethylanthrascene and 9-cyano,10-phenylanthracene-amine systems. ${ }^{22,24}$ In order to obtain information on the relative magnitudes of the different rate constants by temperature effects on exciplex formation for PMOS, PEOS, and PMMS systems, a graph of $\log I_{(\mathrm{PSD}-\mathrm{DMT})^{*}} / I_{(\mathrm{PSD})^{*}}$ was plotted against $1 / \mathrm{T}$ and is given in Figure 6.

The linear region at low temperatures between room temperature and about the glass transition temperature $\left(T_{\mathrm{g}}\right)$ corresponds to the conditions under which the rate constant of radiationless processes $k_{\mathrm{IM}}, k_{\mathrm{IE}}$ and $k_{\mathrm{IEx}}$ increase on increasing the temperature. At high temperatures between $\left(T_{\mathrm{g}}\right)$ and $385 \mathrm{~K}$, the obtained linear region corresponds to the conditions under which the dissociation rate constants of the formed exciplexes via $k_{\mathrm{ExM}} k_{\mathrm{EM}}$ and $k_{\mathrm{IM}}$ becomes higher at higher temperatures. If we take into account the lowering in glass transition temperature $\left(T_{\mathrm{g}}\right)$ that was caused by the presence of the plasticizer DMT, ${ }^{25,26}$ then the turning point between the two regions is not far from the $\left(T_{\mathrm{g}}\right)$ values of the three polymers. Redissolving the heated film gave a reasonably high intensity of exciplex emission, indicating that DMT was not destroyed by heating the solid film. The reason for this behavior may be attributed to the thermal quenching of excimer emission as well as to the possible phase separation between DMT and polymer chain at higher temperatures.

\section{Activation Energy Associated with Exciplex Dissocia- tion}

As can be seen in Figure 6, the thermal effect on solid films of PSD is similar to that observed for polystyrene, ${ }^{27}$ in which two temperature regimes may be distinguished. The first is observed between $293 \mathrm{~K}$ and about glass transition temperature of the DMT doped PSD host. The decrease in $\log I_{(\mathrm{PSD}-\mathrm{DMT})^{*}} / I_{(\mathrm{PSD})^{*}}$ at the higher temperature of this region is attributed to exciplex dissociation, where $k_{\mathrm{EXM}}>\left(k_{\mathrm{FEX}}+k_{\mathrm{IEX}}\right)$. This assumption is supported by the temperature effect on the increase of the polymer fluorescence and a decrease in exciplex fluorescence as in Figure 6. The activation energy of intermolecular exciplex formation has been obtained from the relation $\log \left[I_{(\mathrm{PSD}-\mathrm{DMT})^{*}} /\right.$ $\left.I_{(\mathrm{PSD})^{*}}\right]=-E a / 2.303 \mathrm{RT}$. For more clarification, Arrhenius plots are shown in Figure 7. The activation energy of the exciplex formation in PMPS is $6.1 \mathrm{~kJ}$ compared with $11.4 \mathrm{~kJ}$ for PMOS and $11.7 \mathrm{~kJ}$ for PEOS. The smaller value of PMPS can be attributed partially to the charge transfer character of [PMPS$\mathrm{DMT}^{*}$ system and partially to the fact that fluorescence quenching occurs over a larger distance in this system than in the other systems of PEOS and PMOS. These circumstances result in smaller activation energy for the exciplex formation in PMPS. 


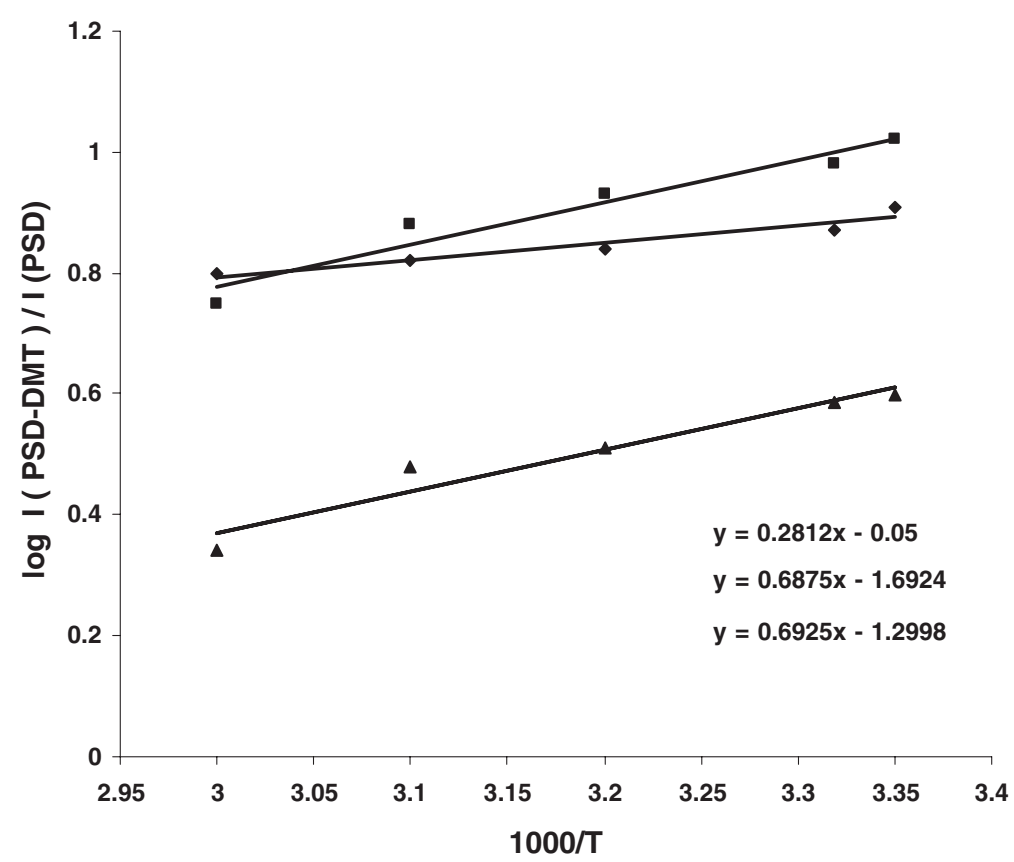

Figure 7. Arrhenius plot for the determination of exciplex activation energies of polystyrene derivatives, $\mathbf{a M P S}+3 \%$ DMT), $\Delta(\mathrm{PEOS}+3 \% \mathrm{DMT})$ and $\bullet$ PMOS $+3 \% \mathrm{DMT})$.

From the obtained results, an increase in temperature of the solid films of the used polymers causes a decrease in both excimer and exciplex emissions. This may be attributed to an increase in chain session which produces polystyryl radicals, ${ }^{27}$ and also to the increase of molecular motion, which results in more molecular collisions and subsequent loss of energy. ${ }^{28}$ The mobility of the formed radicals was found to be affected by the matrix temperature near $\left(T_{\mathrm{g}}\right)$ values, and decreases with increasing temperature. This evidence suggests that the recombination reaction between polystyryl radicals takes place near $\left(T_{\mathrm{g}}\right)$, at which segmental motion begins to occur in the used polymers backbone, and accelerate above this temperature. Accordingly, activation energy values $(\Delta E)$ were expected to vary between temperatures above and below $\left(T_{\mathrm{g}}\right)$ values. The reported data on activation energies for excimer formation were concentrated on the temperature region below glass transition temperature for the used polymers.

Acknowledgment. K. A. acknowledges the financial support offered by the Hashemite University and Abdul Hameed Showman Foundation. We are acknowledging Professor Al-Barqawi and Dr. Yaser al Haj for the useful discussion we had concerning exciplex formation. F. R. acknowledges the Department of Industrial Chemistry at the Institute of Technology, Baghdad, Iraq.

\section{REFERENCES}

1. D. V. O'Conner, L. Chewter, and D. Phillips, J. Phys. Chem., 86, 3400 (1982).

2. H. Masuhara, T. Hino, and N. Mataga, J. Phys. Chem., B, 79, 994 (1975).

3. N. Mataga, T. Okada, and N. Yamamoto, Bull. Chem. Soc. Jpn., 39, 2562 (1966).

4. "Intermolecular Excimers in Organic Molecular Photochemistry," W. Klopffer and J. B. Birks, Ed., Wiley-Interscience, New York, 1973, p 537.

5. C. E. Hoyle and J. E. Juillet, Macromolecules, 11, 221 (1978).

6. K. Iwai, Y. Itoh, and M. Furue Shun-Ichim Nozakura, J. Polym. Sci., Polym. Chem. Ed., 21, 2439 (1983).

7. K. E. Al-Ani, T. I. Tawfiq, and R. K. Ahmed, Iraqi J. Sci., 32, 15 (1991) Photochemistry, 1, Irbid, Jordan, (1989).

8. T. Ishii, T. Hand, and S. Matsunaga, J. Polym. Sci., Polym. Phys. Ed., 17, 11 (1979).

9. S. E. Webber, Peter E. Avots-Avotins, and Michel Deumie, Macromolecules, 14, 110 (1981).

10. M. Guaita, O. Chiantore, and L. Costa, Polym. Degrad. Stab., 12, 315 (1985).

11. A. Guyot, Polym. Degrad. Stab., 15, 219 (1986).

12. I. C. McNeill, M. Zulfiqar, and T. Kousar, Polym. Degrad. Stab., 28, 131 (1990).

13. I. C. McNeill and M. Coskun, Polym. Degrad. Stab., 17, 347 (1987).

14. I. C. McNeill and M. Coskun, Polym. Degrad. Stab., 23, 175 (1989).

15. B. Boinon, D. Ainad-Tabet, and J. P. Montheard, Polym. Degrad. Stab., 28, 197 (1990). 
16. M. Coskun, K. Demirelli, and E. Ozdemir, Polym. Degrad. Stab., 47, 251 (1995).

17. V. V. Zuev, F. Bertini, and G. Audisio, Polym. Degrad. Stab., 71, 213 (2000).

18. K. E. Al-Ani, J. Abhath Alyarmouk, 2, 289 (2001).

19. K. E. Al-Ani, T. Akasheh, and Y. Yousef, Asian J. Chem., 18, (2006) in press.

20. G. E. Johnson, Macromolecules, 13, 145 (1980).

21. L. A. Harrah, J. Chem. Phys., 56, 385 (1972).

22. S. Hirayama, Chem. Phys. Lett., 63, 569 (1979).

23. J. B. Birks, "Photophysics of Aromatic Molecules,"
Wiley-Interscience, New York, 1970, p 303.

24. S. Hirayama, Int. J. Quantum Chem., 19, 254 (1980).

25. P. Smith and E. McGill, J. Polym. Sci., Polym. Phys. Ed., 26, 569 (1988).

26. P. Smith and A. Eisenberg, J. Polym. Sci., Polym. Phys. Ed., 26, 581 (1988).

27. A. Torikai, A. Takeuchi, and K. Fucki, Polym. Degrad. Stab., 14, 213 (1986).

28. G. G. Guilbault, "Practical fluorescence," 2nd ed., Marcel Dekker, Inc., New York, 1990, p 28. 